\title{
Indicador de Rentabilidad de Proyectos: el Valor Actual Neto (VAN) o el Valor Económico Agregado (EVA)
}

\begin{abstract}
RESUMEN
El presente artículo brinda un análisis sobre la rentabilidad de los proyectos de inversión y los indicadores usuales para determinar dicha rentabilidad, enfocándose en el estudio del Valor Económico Agregado y el Valor Actual Neto, herramientas muy utilizadas en el campo de las finanzas, para ello se analizan sus conceptos, interpretaciones y aplicaciones.
\end{abstract}

Palabras clave: Evaluación de proyectos, Valor Económico Agregado, Valor Actual Neto.

Project Performance indicator: Net Present Value (NPV) or Economic Value AdDEd (EVA)

\section{ABSTRACT}

This article provides an analysis of the profitability of investment projects and the usual indicators for determining such return, focusing on the study of Economic Value Added and Net Present Value, used tools in the field of finance, this will analyze concepts, interpretations and applications.

Keywords: Project evaluation, Economic Value Added, Net Present Value.

\section{INTRODUCCIÓN}

Cuando se analiza el proceso de evaluación de proyectos comúnmente se relaciona al uso de indicadores de rentabilidad, usualmente el Valor Actual Neto, la Tasa Interna de Rendimiento (TIR) y en algunos casos se menciona al Valor Económico Agregado (EVA). Por esta razón es importante analizar dichos conceptos para incorporarlos en forma adecuada en el proceso de evaluación de proyectos en el caso que lo amerite.

En nuestro país y en los países de América Latina la evaluación de proyectos se ha ligado a la disciplina de las finanzas, su influencia se destaca en el uso de herramientas con el enfoque financiero, por cierto muy distinto al enfoque económico de los proyectos. Es el caso del flujo de caja que muchas veces se obtiene de los estados financieros y se le denomina flujo de fondos. Así también el EVA es una herramienta netamente financiera que se utiliza en forma complementaria con el VAN.

\section{EVALUACIÓN DE PROYECTOS}

En el campo empresarial las decisiones de inversión son muy importantes porque son el medio para implementar las estrategias y lograr los objetivos que se han propuesto. El documento básico para el análisis de la decisión de inversión es el proyecto de inversión. La evaluación de proyectos permite medir las bondades de la inversión desde el punto de vista económico, por ello se estiman los probables ingresos y costos en un horizonte de tiempo, la comparación de los valores genera un conjunto de indicadores que muestran la rentabilidad y determinan la conveniencia de ejecutar el proyecto.

* Ingeniero Industrial UNMSM, Profesor en la Facultad de Ingeniería Industrial, Departamento Académico de Producción y Gestión Industrial de la UNMSM. E-mail: cieandia@hotmail.com 


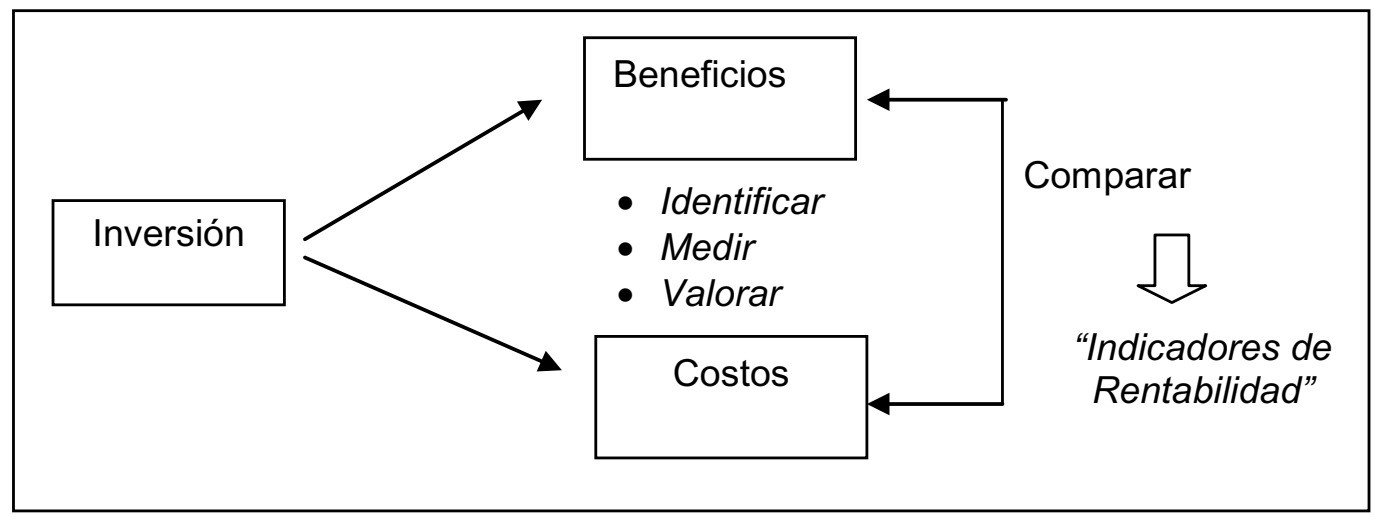

Figura N. ${ }^{\circ}$ 1. El Proceso de Evaluación de proyectos.

\section{Valor Económico Agregado}

EI EVA es una medida que refleja en términos absolutos el desempeño global de una empresa basado en la generación de valor, a diferencia de los indicadores de rentabilidad que muestran información parcial. Por ejemplo, la rentabilidad sobre la inversión (ROI) nos muestra la generación de utilidad con respecto a lo invertido y considera el costo de financiamiento de sus pasivos, pero debe ser comparada con el costo de oportunidad del dinero de los inversionistas para conocer si se cubre sus expectativas; mientras el EVA incorpora en su análisis todos los aspectos anteriores.

En toda actividad empresarial se utilizan recursos, los que tienen un costo; para que el negocio sea viable se deben generar ingresos que cubran todos los costos incluyendo el financiamiento, si es así se está generando valor para la empresa, de lo contrario se destruye valor, porque se tienen que utilizar recursos de otras fuentes para cubrir dicho déficit. Figura N. ${ }^{\circ}$.
Por la sencillez de su concepto es fácil de aplicar, surge de comparar la rentabilidad obtenida por una empresa con el costo de los recursos utilizados para obtener dicha rentabilidad. Matemáticamente se expresa de la siguiente manera:

\section{EVA $=/$ Rentabilidad - Costo de Capital $) *$ Capital}

Es importante aclarar los conceptos utilizados, se debe entender como "capital" a todos los recursos utilizados por la empresa para generar sus actividades (pasivos y patrimonio) y el costo de capital $\left(c^{*}\right)$ representa el valor del costo de esos recursos. La ecuación también se puede expresar de la siguiente forma:

$$
\text { EVA }=r^{*} \text { Capital }-c * \text { Capital }
$$

Ahora basándose en los indicadores financieros, la rentabilidad se puede expresar como la utilidad antes de gastos financieros y después de impuestos (UAIDI) (no se incluye los gastos financieros para no duplicarlo en el costo de capital) respecto al capital.

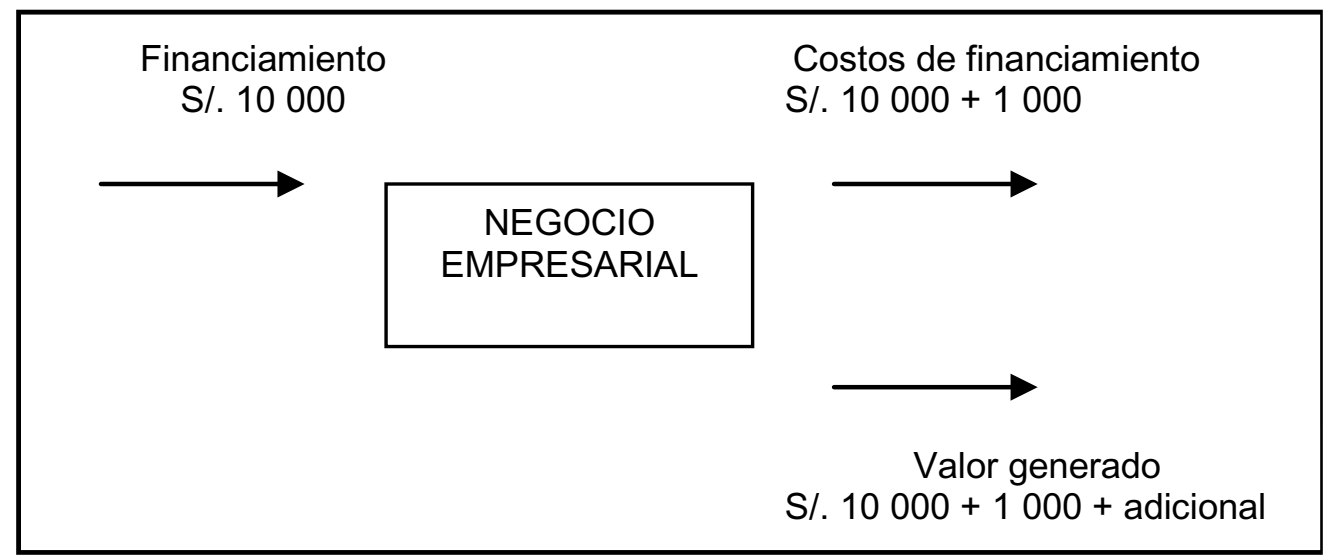

Figura $\mathbf{N}^{\circ}{ }^{\circ}$ 2. El Valor en los Negocios. 


$$
r=\frac{U A I D I}{\text { Capital }}
$$

Entonces si reemplazamos la ecuación quedaría así:

$$
E V A=U A I D I-c^{*} \text { Capital }
$$

Es decir, para hallar el EVA se debe restar a la utilidad antes de interés y después de impuestos UAIDI el costo de capital expresado en términos monetarios.

Por ejemplo, si una empresa tiene una UAIDI de S/. 50 000, un capital de S/. 200000 cuyo costo es 15\%, EVA sería:

$$
\begin{gathered}
E V A=50000-0.15 * 200000 \\
E V A=20000
\end{gathered}
$$

Es decir, la empresa ha generado una riqueza de S/. 20000 en ese periodo. Como se puede apreciar el EVA es el resultado obtenido una vez que se han cubierto todos los gastos y satisfecho una rentabilidad mínima esperada por parte de los accionistas.

\section{Caso}

Se tiene la siguiente información de los Estados Financieros, el procedimiento para determinar el EVA es el siguiente:

a. Hallar la utilidad antes de intereses y después de impuestos (UAIDI)

Tabla N. ${ }^{0}$ 1. Estado de Ganancias y Pérdidas.

\begin{tabular}{|lr|}
\hline DETALLE DE LA CUENTA & 20X9 \\
\hline Ventas netas & 77584 \\
Costo de ventas & 45052 \\
Utilidad bruta & 32532 \\
Gastos operativos & 26057 \\
Utilidad operativa & 6475 \\
Otros ingresos & 4589 \\
Intereses pagados & 2158 \\
Utilidad antes de impuestos & 8906 \\
Impuesto a la renta & 2672 \\
Utilidad neta & 6234 \\
\hline
\end{tabular}

Una forma práctica de hallar la UAIDI es sumando a la utilidad neta los intereses pagados. Por lógica, lo que se busca es cuánto ha generado la empresa sin considerar los intereses.

$$
\begin{aligned}
& \text { UAIDI = Utilidad neta }+ \text { Intereses } \\
& \text { UAIDI = } 6234+2158 \\
& \text { UAIDI = } 8392
\end{aligned}
$$

b. Determinar el costo de capital

Para generar dicha utilidad la empresa ha utilizado bienes y recursos, contablemente son los activos, ellos son financiados por el pasivo y el patrimonio, cada uno con sus respectivos costos, por lo que es necesario determinar su costo promedio.

Tabla N. ${ }^{\circ}$ 2. Costo de Capital.

\begin{tabular}{|lccr|}
\hline & $20 \times 9$ & $\%$ & Costo \\
\hline TOTAL PASIVO CORRIENTE & 18484 & 0,2739 & $12 \%$ \\
TOTAL PASIVO NO CORRIENTE & 11843 & 0,1755 & $10 \%$ \\
TOTAL PASIVO & 30327 & & \\
TOTAL PATRIMONIO & 37152 & 0,5506 & $9 \%$ \\
\hline TOTAL PASIVO Y PATRIMONIO & $\mathbf{6 7 4 7 9}$ & $\mathbf{1 , 0 0}$ & \\
\hline
\end{tabular}

Como se presentan varias fuentes de financiamiento se utiliza el costo promedio ponderado de capital - CPPC.

$\mathrm{CPPC}=\mathrm{i}_{\mathrm{p} *}($ pasivo/activo $) *(1-\mathrm{t})+\mathrm{i}_{\mathrm{pt}}$ * (patrimonio/activo)

Donde:

$\mathrm{i}_{\mathrm{p}}$ : costo del pasivo

$\mathrm{t}$ : tasa del impuesto a la renta $(30 \%)$

$\mathrm{i}_{\mathrm{pt}}$ : costo del patrimonio

El factor $(1-t)$ es para determinar el costo neto de la deuda, considerando los efectos del ahorro por el menor pago de impuesto a la renta y solo se aplica al pasivo. En este caso no se utiliza porque se estaría duplicando dicho beneficio inmerso ya en la rentabilidad.

$C P P C=\frac{18484}{67479} * 0.12+\frac{11843}{67479} * 0.10+\frac{37152}{67479} * 0.09$

$C P P C=9.9 \%$

c. Calcular el EVA

$$
\begin{aligned}
& E V A=U A I D I-c * \text { Capital } \\
& E V A=8392-0.099 * 67479 \\
& E V A=1650
\end{aligned}
$$

\section{EI Valor Actual Neto (VAN)}

EI VAN es un indicador que forma parte del análisis beneficio costo, es decir, cuando se aplica en aque- 
llos casos en que los beneficios de una inversión compense a los costos.

EI VAN es un indicador que muestra la riqueza adicional que genera un proyecto luego de cubrir todos sus costos en un horizonte determinado de tiempo, es decir, cuando se analiza una inversión, lo mínimo que se debe obtener es: cubrir sus costos.

Desde el punto de vista matemático el VAN acumula los beneficios y costos en el periodo cero. Por ejemplo se tiene una inversión de S/. 50000 que genera beneficios netos (ingresos menos costos operativos, financieros) de S/. 20000 el primer año, de S/. 30000 el segundo y tercero; considerando una tasa mínima de rendimiento de $12 \%$ determine el VAN.

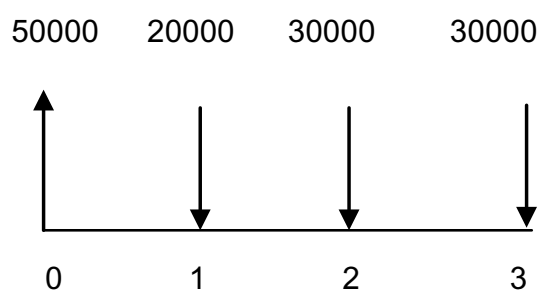

$$
\begin{aligned}
& \text { Se halla el VAN } \\
& \begin{array}{l}
\text { VAN }(12 \%)=20000 /(1,12)+30000 /(1,12)^{2}+30 \\
000 /(1,12)^{3}-50000 \\
\text { VAN }(12 \%)=13126
\end{array}
\end{aligned}
$$

La interpretación es la siguiente: El proyecto cubre todos sus costos, así como la tasa mínima del $12 \%$ requerida, además de ello genera una riqueza adicional de S/. 13 126, es decir, el valor generado.

\section{El Valor Económico Agregado (EVA) y el Valor Actual Neto (VAN)}

El Valor Actual Neto tiene como concepto medir la generación de riqueza cuando se realiza una inversión mediante un proyecto, es decir, es similar al enfoque lógico del EVA, ambos miden la generación de riqueza o valor, la diferencia se encuentra en su aplicación, el VAN mide el valor de un proyecto en un horizonte intertemporal (horizonte de tiempo) mientras que el EVA mide el valor en un periodo de tiempo determinado.

Además, el VAN es un indicador de rentabilidad en el análisis de las inversiones y su enfoque es económico, mientras que el EVA es un indicador de gestión financiera asociado a los aspectos contables, por lo tanto, el primero se debe usar en evaluación de proyectos y el segundo en el análisis financiero.

\section{CONCLUSIONES}

1. En el análisis de inversiones se tiene un conjunto de indicadores, entre ellos tenemos el Valor Actual Neto que brinda la riqueza adicional generada por un proyecto de inversión.

2. En el campo de las finanzas se utiliza el Valor Económico Agregado para medir el valor que genera una empresa en un determinado periodo.

3. Tanto el VAN como el EVA miden la generación de valor, uno en el campo de los proyectos y otro en el campo financiero.

\section{REFERENCIAS BIBLIOGRÁFICAS}

[1] Andía Valencia, W. (2010). Manual Práctico de Finanzas Empresariales, Primera edición. El Saber Editores. Perú.

[2] Andía Valencia, W. (2010). Gerencia de Proyectos Sociales y de Inversión Social. 1ra edición. El Saber Editores. Perú.

[3] Andía Valencia, W (2009). Proyectos de Inversión. Segunda edición. El Saber Editores. Perú.

[4] Castro, R. y Mokate K. (1994). Evaluación económica de proyectos de inversión, Facultad de Economía, Universidad de los Andes, Colombia. 